\title{
A CROSS SECTION THEOREM AND AN APPLICATION TO $C^{*}$-ALGEBRAS
}

\author{
ROBERT R. KALLMAN ${ }^{1}$ AND R. D. MAULDIN
}

\begin{abstract}
The purpose of this note is to prove a cross section theorem for certain equivalence relations on Borel subsets of a Polish space. This theorem is then applied to show that cross sections always exist on countably separated Borel subsets of the dual of a separable $C^{*}$-algebra.
\end{abstract}

See Auslander-Moore [2], Bourbaki [3], Kuratowski [9], and Mackey [12] for the main results and notation in Polish set theory used in this paper.

The main result of this note is the following theorem.

Theorem 1. Let $B$ be a Borel subset of the Polish space $X$. Let $R$ be an equivalence relation on $B$ such that each $R$-equivalence class is both $a G_{\delta}$ and an $F_{\sigma}$ in $X$, and such that the $R$-saturation of each relatively open subset of $B$ is Borel. Then the quotient Borel space $B / R$ is standard, and there is a Borel cross section $f: B / R \rightarrow B$ for $R$.

Notice that if the $R$-saturation of each relatively closed subset of $B$ is Borel, then the $R$-saturation of each relatively open subset of $B$ is Borel, for each relatively open subset of $B$ is the countable union of relatively closed sets.

A number of preliminary lemmas are proved first.

LEMMA 2. Let $(Y, d)$ be a separable metric space and let $R$ be an equivalence relation on $Y$ such that the $R$-saturation of each open set is Borel. Then there is a Borel set $S$ whose intersection with each $R$-equivalence class which is complete with respect to $d$ is nonempty, and whose intersection with each $R$-equivalence class is at most one point.

Proof. By the proofs (but not the statements) of Theorem 4, p. 206, Bourbaki [3] and Lemme 2, p. 279, Dixmier [4], there exists a decreasing sequence of Borel subsets of $Y$, say $S_{n}$, so that $S_{n} \cap R(y) \neq \varnothing$, diameter $\left(S_{n}\right.$ $\cap R(y)) \rightarrow 0$, and $\cap_{n \geqslant 1}\left(S_{n} \cap R(y)\right)=\bigcap_{n \geqslant 1}\left(\left(\overline{S_{n} \cap R(y)}\right) \cap R(y)\right)$ for each $y$ in $Y$. Let $S=\cap_{n \geqslant 1} S_{n}$. $S$ is a Borel subset of $Y$, the intersection of $S$ with each complete $R$-equivalence class is nonempty, and the intersection of $S$ with each $R$-equivalence class is at most one point. Q.E.D.

Lemma 3. Let $Y$ be a Polish space and $D$ a subset of $Y$ which is both $a G_{\delta}$ and

Received by the editors November 15, 1976 and, in revised form, July 18, 1977.

AMS (MOS) subject classifications (1970). Primary 28A05, 46L05; Secondary 04A15, 54C65. $K e y$ words and phrases. Quotient Borel space, Borel cross section, $C^{*}$-algebra.

${ }^{1}$ Supported in part by NSF Grant MPS73-08628.

๑ American Mathematical Society 1978 
an $F_{\sigma}$. Then there is an open set $V$ in $Y$ so that $D \cap V$ is nonempty and $D \cap V$ is closed in $V$.

Proof. We may assume that $\bar{D}=Y$. Since $D$ is a $G_{\delta}, Y-D$ is a countable union of closed sets. None of these closed sets has an interior, for $D$ is dense in $Y$. But $D$ is a countable union of closed sets. The Baire category theorem implies that one of these closed sets has an interior. Hence, there exists an open set $V$ so that $V$ is contained in $D$. Q.E.D.

LEMMA 4. Let $X$ and $R$ be as in Theorem 1. Then $X / R$ is countably separated.

Proof. Let $V_{m}(m \geqslant 1)$ be a basis for the topology of $X$. Then the $R\left(V_{m}\right)$ $(m \geqslant 1)$ are Borel sets which separate the $R$-equivalence classes. To see this, let $a$ and $b$ be elements of $X$ so that $R(a)$ and $R(b)$ are disjoint. If $R(b)$ is not contained in $\overline{R(a)}$, there exists a $c$ in $R(b)$ and a positive integer $m$ so that $c$ is in $V_{m}$ and $V_{m} \cap \overline{R(a)}=\varnothing$. But then $V_{m} \cap R(a)=\varnothing$, and so $R\left(V_{m}\right) \cap$ $R(a)$ is empty. Hence, $R(b)$ is contained in $R\left(V_{m}\right)$ and $R(a)$ is contained in $X-R\left(V_{m}\right)$. So we may assume that $R(b)$ is contained in $\overline{R(a)}$. It follows from Lemma 3 that there is an integer $m$ so that $R(a) \cap V_{m}$ is nonempty and $R(a) \cap V_{m}=\overline{R(a)} \cap V_{m}$. But then $R\left(V_{m}\right) \cap R(b)$ is empty. If not, there exists a $c$ in $R(b) \cap V_{m} \subseteq \overline{R(a)} \cap V_{m}=R(a) \cap V_{m}$. Hence, $R(b)=R(c)=$ $R(a)$. Contradiction. Hence, $R(a)$ is contained in $R\left(V_{m}\right)$ and $R(b)$ is contained in $X-R\left(V_{m}\right)$. Q.E.D.

Proof of THeOrem 1. If $V$ is an open subset of $X$ and $U=B \cap V, R$ defines an equivalence relation $R_{U}$ on $U$ by $R_{U}(b)=R(b) \cap U$. The $R_{U^{-}}$ saturation of any open set is Borel. Now $V$ itself is a Polish space, and each $R_{U}$-equivalence class is both a $G_{\delta}$ and an $F_{\sigma}$ in $V$. Hence, by Lemma 2 , there is a Borel set $S$ which intersects each $R_{U}$-equivalence class in at most one point, and which intersects each $R_{U}$-equivalence class which is closed in $V$ in exactly one point. Let $V_{m}(m \geqslant 1)$ be a basis for the topology of $X$. For each $V_{m}$, let $S_{m}$ be a corresponding $S$, and let $S^{\prime}=\cup_{m>1} S_{m} \cdot S^{\prime}$ is a Borel subset of $X . S^{\prime}$ intersects each $R$-equivalence class in at most countably many points. Furthermore, $S^{\prime}$ intersects each $R$-equivalence class in at least one point by Lemma $3 . X / R$ is countably separated, and therefore is Borel isomorphic to an analytic subset of $[0,1]$ by Proposition 2.9 , p. 8, of Auslander-Moore [2]. Let $g: S^{\prime} \rightarrow X / R$ be the natural surjective Borel mapping. The graph of $g$, say $C$, is a Borel subset of $S^{\prime} \times[0,1]$. Horizontal sections of $C$ are at most countable. Hence, theorems of $\mathrm{S}$. Braun and $\mathrm{N}$. Luzin (see 42.4.5, p. 378, and 42.5.3, p. 381, of Hahn [8]) show that the horizontal projection of $C$, namely $X / R$, is standard and that there exists a Borel subset $S^{\prime \prime}$ of $S^{\prime}$ so that $g \mid S^{\prime \prime}$ is a bijection onto $X / R$. Let $f=\left(g \mid S^{\prime \prime}\right)^{-1}$. $f$ is a Borel mapping by Souslin's theorem. Q.E.D.

See Dixmier [5] for most of the notation and results on $C^{*}$-algebras used in this note. The Borel structure on the dual of a $C^{*}$-algebra is that generated by the hull-kernel topology. The following corollary might be a useful tool in 
proving local versions of known theorems in $C^{*}$-algebrase and group representations (see, for instance, Moore's appendix to Auslander and Kostant [1]).

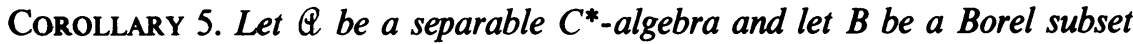
of $\hat{\mathbb{Q}}$ whose relative Borel structure separates points. Then $B$ is standard, and there is a Borel cross section $f: B \rightarrow \operatorname{Irr}(\mathbb{Q})$.

Proof. Let $p: \hat{\pi} \rightarrow \operatorname{kernel}(\hat{\pi}), \hat{\mathbb{Q}} \rightarrow \operatorname{Prim}(\mathbb{Q})$, be the natural open mapping. From the definition of the topology of $\hat{\mathcal{Q}}, U$ is open in $\hat{\mathcal{Q}}$ if and only if $p(U)$ is open in $\operatorname{Prim}(\mathscr{Q})$, in which case $U=p^{-1}(p(U))$. Now consider the set $\mathcal{S}$ of all subsets $B$ of $\hat{Q}$ such that $B=p^{-1}(p(B))$. $\mathcal{S}$ is clearly closed under countable unions, and $\mathcal{S}$ is closed under complements since $p$ is surjective. Since $\mathcal{S}$ contains the open subsets of $\hat{\mathcal{Q}}, \mathcal{S}$ therefore contains all Borel subsets of $\hat{\mathcal{Q}}$. Therefore, $B$ is a Borel subset of $\hat{\mathcal{Q}}$ if and only if $p(B)$ is a Borel subset of Prim $(\mathscr{Q})$. Hence, if $B$ is a Borel subset of $\hat{Q}$, and if the relative Borel structure on $B$ separates points, then $p$ is one-to-one on $B$, and $B$ and $p(B)$ are Borel isomorphic. But Prim( $(Q)$ is a standard Borel space by Theorem 2.4 of Effros [7]. Hence, $p(B)$, and therefore $B$, are standard Borel spaces.

Let $q: \operatorname{Irr}(\mathscr{Q}) \rightarrow \hat{\mathbb{Q}}$ be the natural continuous open mapping. As $\operatorname{Prim}(\mathscr{Q})$ is $T_{0}$ with a countable basis for its topology, each point of $\operatorname{Prim}(\mathbb{Q})$ is the intersection of a closed set and a $G_{\delta}$. Hence, $q^{-1}(b)=(p \circ q)^{-1}(p(b))$ is a $G_{\delta}$ in $\operatorname{Irr}(Q)$ for all $b$ in $B$. Each $q^{-1}(b)$ is also an $F_{o}$ by Lemma 2.7 and Lemma 4.1 of Effros [6]. Let $R$ be the equivalence relation on $q^{-1}(B)$ given by point inverses under $q$. Each $R$-equivalence class is both a $G_{\delta}$ and an $F_{\sigma}$ in $\operatorname{Irr}(\mathscr{Q})$. The $R$-saturation of a relatively open subset of $q^{-1}(B)$ is again relatively open, and therefore Borel, since $q \mid q^{-1}(B)$ is open onto $B$. Hence, Theorem 1 and Souslin's theorem show that $q^{-1}(B) / R$ and $B$ are Borel isomorphic, and there is a cross section $f: B \rightarrow \operatorname{Irr}(\mathbb{Q})$. Q.E.D.

The following corollary has some applications. Consider the following setup. Let $X$ be a standard Borel space, $Y$ a Polish space, and $R$ an equivalence relation on $Y$ such that the $R$-saturation of open sets is Borel. $R$ gives rise to an equivalence relation $R^{\prime}$ on $X \times Y$ by $R^{\prime}(x, y)=\{x\} \times$ $R(y)$.

Corollary 6. Let $B$ be a Borel subset of $X \times Y$ which is saturated with respect to $R^{\prime}$. Suppose that each $R^{\prime}$-equivalence class contained in $B$ is, viewed as a subset of $Y$, both $a G_{\delta}$ and an $F_{\sigma}$. Then $B / R^{\prime}$ is standard, and there exists a Borel cross section $f: B / R^{\prime} \rightarrow B$ for $R^{\prime}$.

Proof. There exists a Polish space $Z$ and a one-to-one Borel mapping $p$ : $Z \rightarrow X$. Let $g:(z, y) \rightarrow(p(z), y), Z \times Y \rightarrow X \times Y$. Let $R^{\prime \prime}=g^{-1}\left(R^{\prime}\right)$ and $B^{\prime}=g^{-1}(B)$. Each $R^{\prime \prime}$-equivalence class contained in $B^{\prime}$ is both a $G_{\delta}$ and an $F_{\sigma}$ since each $R^{\prime}$-equivalence class in $B$ is, viewed as a subset of $Y$, a $G_{\delta}$ and an $F_{\sigma}$, and since the vertical sections of $Z \times Y$ are closed. The $R^{\prime \prime}$-saturation of an open set in $Z \times Y$ is Borel. It suffices to prove this for open rectangles. Let $U \times V$ be open in $Z \times Y$, where $U$ is open in $Z$ and $V$ is open in $Y$. But 


$$
R^{\prime \prime}(U \times V)=g^{-1}\left(R^{\prime}(p(U) \times V)\right)=g^{-1}(p(U) \times R(V)),
$$

which certainly is Borel in $Z \times Y$. Hence, by Theorem $1, B^{\prime} / R^{\prime \prime}$ is standard, and there exists a Borel cross section $f^{\prime}: B^{\prime} / R^{\prime \prime} \rightarrow B^{\prime}$. Choose a sequence $B_{n}^{\prime}$ $(n \geqslant 1)$ of $R^{\prime \prime}$-saturated Borel subsets of $B^{\prime}$ which separate the $R^{\prime \prime}$-equivalence classes. Then the $g\left(B_{n}^{\prime}\right)(n \geqslant 1)$ are $R^{\prime}$-saturated Borel subsets of $B$ which separate the $R^{\prime}$-equivalence classes. Hence, $B / R^{\prime}$ is countably separated. Furthermore, $g\left(f^{\prime}\left(B^{\prime} / R^{\prime \prime}\right)\right)$ is a Borel transversal for the $R^{\prime}$-equivalence classes of $B$. Let $h: g\left(f^{\prime}\left(B^{\prime} / R^{\prime \prime}\right)\right) \rightarrow B / R^{\prime}$ be the natural one-to-one Borel mapping. Then $B / R^{\prime}$ is standard by Souslin's theorem, and $f=h^{-1}$ : $B / R^{\prime} \rightarrow B$ is a Borel cross section for $R^{\prime}$. Q.E.D.

The following examples help to clarify the hypotheses of Theorem 1 .

EXAMPLE 7. Note that Theorem 1 may fail if each $R$-equivalence class is only required to be an $F_{\sigma}$ set, even if the $R$-saturation of each open set is open and $B / R$ is metrizable. This follows from the fact that if $A$ is an analytic nonborelian subset of $J$, the irrational numbers, then there is a Borel subset $B$ of $J \times J$ such that the projection map restricted to $B$ is open and projects $B$ onto $A$. Also, each vertical section of $B$ may be taken to be an $F_{\sigma}$ subset of (see Taimanov [11]).

EXAMPLE 8. There is a Borel subset $B$ of $J \times J$ such that each vertical section of $B$ is an $F_{\sigma}$ subset of $J$, the projection $\pi$ onto the first axis, restricted to $B$, is open, $\pi(B)=J$, and yet there is no Borel cross section (in this case, there is no Borel uniformization). Recall that if $E$ is a subset of $X \times Y$, then a uniformization of $E$ is a subset $F$ of $E$ such that $E_{x} \neq \varnothing$ if and only if $F_{x}$ consists of exactly one point, where $E_{x}=[y \mid(x, y)$ is in $E]$.

First, let $M$ be a Borel subset of $J \times J$ such that $\pi(M)=J, M$ has no Borel uniformization, and each vertical section of $M$ is closed. The existence of such an $M$ can be seen as follows. Let $C_{1}$ and $C_{2}$ be disjoint coanalytic subsets of $J$ which are not Borel separable (see Sierpinski [10] for the existence of these C's). Let $A_{1}=J-C_{1}$ and $A_{2}=J-C_{2} . A_{1}$ and $A_{2}$ are analytic sets whose union is $J$. Let $M_{i}$ be a closed subset of $J \times J$ which projects onto $A_{i}(i=1,2)$. Let $M$ be the Borel set which is the union of $M_{1}$ and $M_{2}$. If $\Gamma$ were a Borel uniformization of $M$, then $D=\pi\left(\Gamma \cap\left(M_{1}-M_{2}\right)\right)$ would be a Borel subset of $J$ which contains $C_{2}$ and has empty intersection with $C_{1}$. Thus, $M$ has no Borel uniformization. This argument for the existence of $M$ is due to D. Blackwell.

Identify $J$ with $N^{N}$. Let $h_{n_{1}} \ldots n_{k}$ be a homeomorphism of $J$ onto $J\left(n_{1}, \ldots, n_{k}\right)=\left[z \mid z\right.$ is in $J$ and $\left.z_{i}=n_{i}(1 \leqslant i \leqslant k)\right]$, and let $T_{n_{1}} \cdots n_{k}:(x, z)$ $\rightarrow\left(x, h_{n_{1} \cdots n_{k}}(z)\right), J \times J \rightarrow J \times J$. Let $B=\cup T_{n_{1} \cdots n_{k}}(M)$. Then $B$ is a Borel subset of $J \times J, \pi \mid B$ is open, $\pi(B)=J$, and each vertical section of $B$ is an $F_{\sigma}$. If $\Gamma$ were a Borel uniformization for $B$, then $C=\cup_{n} T_{n}^{-1}((\Gamma \cap$ $\left.T_{n}(M)\right)-\cup_{k<n} T_{k}(M)$ ) would be a Borel uniformization of $M$. Here $k$ and $n$ denote finite multi-indices and $<$ is the usual lexicographic order.

Suppose that $B$ is a Borel subset of a Polish space $X, R$ is an equivalence relation on $B$ such that each equivalence class is a $G_{\delta}$ in $X$, and such that the 
saturation of relatively open sets is Borel. D. Miller has pointed out to the authors that Lemmas 3 and 4 may be altered slightly to prove that $B / R$ is countably separated. Let $V_{m}(m \geqslant 1)$ be as in Lemma 4 . We claim that the $R\left(V_{m}\right)(m \geqslant 1)$ separate the $R$-equivalence classes. Let $a$ and $b$ be in $X$ so that $R(a)$ and $R(b)$ are disjoint. If $R(b)$ is not contained in $\overline{R(a)}$, proceed as in Lemma 4. So suppose that $R(b)$ is contained in $\overline{R(a)}$. By a symmetric argument we may assume that $R(a)$ is contained in $\overline{R(b)}$. Thus, we may assume that $\overline{R(a)}=\overline{R(b)}$. But $R(a)$, being a $G_{\delta}$, is comeager in $\overline{R(a)}$, and $R(b)$, being a $G_{\delta}$, is comeager in $R(b)$. Hence, $R(a) \cap R(b)$ is nonempty, a contradiction. The following questions remain. Is $B / R$ standard? Even if $B / R$ is standard, is there a cross section? The authors do not know the answers to these questions even if $R$ is an open equivalence relation and $B / R$ is metrizable. Note that if the last question has an affirmative answer, then there is a natural Borel cross section from $\operatorname{Prim}(\mathbb{Q}) \rightarrow \operatorname{Irr}(\mathscr{Q})$.

\section{BIBLIOGRAPHY}

1. L. Auslander and B. Kostant, Polarization and unitary representations of solvable Lie groups, Invent. Math. 14 (1971), 255-354.

2. L. Auslander and C. C. Moore, Unitary representations of solvable Lie groups, Mem Amer. Math. Soc., no. 62 (1966).

3. N. Bourbaki, General topology. II, Hermann, Paris, 1966.

4. J. Dixmier, Dual et quasi-dual d'une algèbre de Banach involutive, Trans. Amer. Math. Soc. 104 (1962), 278-283.

5. L Les $C^{*}$-algebres et leurs representations, Gauthier-Villars, Paris, 1969.

6. E. G. Effros, Transformation groups and $C^{*}$-algebras, Ann. of Math. 81 (1965), 38-55.

7.,$A$ decomposition theory for representations of $C^{*}$-algebras, Trans. Amer. Math. Soc. 107 (1963), 83-106.

8. H. Hahn, Reelle Funktionen, Akad.-Verlag, Leipzig, 1932.

9. C. Kuratowski, Topology. I, Academic Press, New York, 1966.

10. W. Sierpinski, Sur deux complémentaires analytiques non separables, Fund. Math. 17 (1931), 296-297.

11. A. D. Taimanov, On open images of Borel sets, Mat. Sb. 37 (1955), 293-300.

12. G. W. Mackey, Borel structure in groups and their duals, Trans. Amer. Math. Soc. 85 (1957), $134-165$.

Department of Mathematics, University of Florida, Gainesville, Florida 32611 